University of Wollongong

Research Online

Faculty of Engineering and Information

Faculty of Engineering and Information

Sciences - Papers: Part A

Sciences

2016

\title{
An implementation of discrete electron transport models for gold in the Geant4 simulation toolkit
}

D Sakata

University of Bordeaux

Sebastien Incerti

University of Bordeaux, incerti@cenbg.in2p3.fr

M Bordage

University of Toulouse

N Lampe

Universite Blaise Pascal

Susumu Okada

KEK

See next page for additional authors

Follow this and additional works at: https://ro.uow.edu.au/eispapers

Part of the Engineering Commons, and the Science and Technology Studies Commons

Research Online is the open access institutional repository for the University of Wollongong. For further information contact the UOW Library: research-pubs@uow.edu.au 


\title{
An implementation of discrete electron transport models for gold in the Geant4 simulation toolkit
}

\author{
Abstract \\ Gold nanoparticle (GNP) boosted radiation therapy can enhance the biological effectiveness of radiation \\ treatments by increasing the quantity of direct and indirect radiation-induced cellular damage. As the \\ physical effects of GNP boosted radiotherapy occur across energy scales that descend down to $10 \mathrm{eV}$, \\ Monte Carlo simulations require discrete physics models down to these very low energies in order to \\ avoid underestimating the absorbed dose and secondary particle generation. Discrete physics models for \\ electron transportation down to $10 \mathrm{eV}$ have been implemented within the Geant4-DNA low energy \\ extension of Geant4. Such models allow the investigation of GNP effects at the nanoscale. At low \\ energies, the new models have better agreement with experimental data on the backscattering \\ coefficient, and they show similar performance for transmission coefficient data as the Livermore and \\ Penelope models already implemented in Geant4. These new models are applicable in simulations \\ focussed towards estimating the relative biological effectiveness of radiation in GNP boosted \\ radiotherapy applications with photon and electron radiation sources.
}

\section{Keywords}

simulation, geant4, gold, toolkit, models, implementation, transport, electron, discrete

Disciplines

Engineering | Science and Technology Studies

\section{Publication Details}

Sakata, D., Incerti, S., Bordage, M. C., Lampe, N., Okada, S., Emfietzoglou, D., Kyriakou, I., Murakami, K., Sasaki, T., Tran, H., Guatelli, S. \& Ivantchenko, V. N. (2016). An implementation of discrete electron transport models for gold in the Geant4 simulation toolkit. Journal of Applied Physics, 120 (24), 244901-1-244901-7.

\section{Authors}

D Sakata, Sebastien Incerti, M Bordage, N Lampe, Susumu Okada, Dimitris Emfietzoglou, loanna Kyriakou, K Murakami, Takashi Sasaki, H Tran, Susanna Guatelli, and V Ivantchenko 


\section{An implementation of discrete electron transport models for gold in the Geant4 simulation toolkit}

D. Sakata, S. Incerti, M. C. Bordage, N. Lampe, S. Okada, D. Emfietzoglou, I. Kyriakou, K. Murakami, T. Sasaki,

H. Tran, S. Guatelli, and V. N. Ivantchenko

Citation: J. Appl. Phys. 120, 244901 (2016); doi: 10.1063/1.4972191

View online: http://dx.doi.org/10.1063/1.4972191

View Table of Contents: http://aip.scitation.org/toc/jap/120/24

Published by the American Institute of Physics

\section{Articles you may be interested in}

Quasi-static major and minor strain-stress loops in textured polycrystalline Fe81.6Ga18.4 Galfenol

J. Appl. Phys. 120, 243901243901 (2016); 10.1063/1.4972479

Ignition and monitoring technique for plasma processing of multicell superconducting radio-frequency cavities J. Appl. Phys. 120, 243301243301 (2016); 10.1063/1.4972838

Current induced magnetization dynamics and magnetization switching in superconducting ferromagnetic hybrid ( F $|\mathrm{S}| \mathrm{F})$ structures

J. Appl. Phys. 120, 243902243902 (2016); 10.1063/1.4972959

Ponderomotive convection in water induced by a CW laser

J. Appl. Phys. 120, 244902244902 (2016); 10.1063/1.4972969

\section{A.P $\mid \begin{aligned} & \text { Journal of } \\ & \text { Applied Physics }\end{aligned}$}

INTRODUCING INVITED PERSPECTIVES

Ultrafast magnetism and $\mathrm{THz}$ spintronics

Authors: Jakob Walowski and Markus Münzenberg 


\title{
An implementation of discrete electron transport models for gold in the Geant4 simulation toolkit
}

\author{
D. Sakata, ${ }^{1,2, a)}$ S. Incerti, ${ }^{1,2}$ M. C. Bordage, ${ }^{3,4}$ N. Lampe, ${ }^{2,5}$ S. Okada, ${ }^{6}$ D. Emfietzoglou, ${ }^{7}$ \\ I. Kyriakou, ${ }^{7}$ K. Murakami, ${ }^{6}$ T. Sasaki, ${ }^{6}$ H. Tran, ${ }^{8}$ S. Guatelli, ${ }^{9}, 10$ and V. N. Ivantchenko ${ }^{11}$ \\ ${ }^{1}$ University of Bordeaux/CENBG, Bordeaux, France \\ ${ }^{2}$ CNRS/IN2P3/CENBG, Bordeaux, France \\ ${ }^{3}$ CRCTIINSERM, Toulouse, France \\ ${ }^{4}$ University of Toulouse III-Paul Sabatier, Toulouse, France \\ ${ }^{5}$ Laboratoire de Physique Corpusculaire, Université Clermont Auvergne, Université Blaise Pascal, \\ CNRS/IN2P3, Clermont-Ferrand, France \\ ${ }^{6} \mathrm{KEK}$, Tsukuba, Japan \\ ${ }^{7}$ Medical Physics Laboratory, University of Ioannina Medical School, Ioannina, Greece \\ ${ }^{8}$ Irfu, CEA, Université Paris-Saclay, 91191 Gif-sur-Yvette, France \\ ${ }^{9}$ Centre For Medical Radiation Physics, University of Wollongong, Wollongong, Australia \\ ${ }^{10}$ Illawarra Health and Medical Research Institute, University of Wollongong, NSW, Australia \\ ${ }^{11}$ Geant4 Associates International Ltd, Hebden Bridge, United Kingdom
}

(Received 3 November 2016; accepted 1 December 2016; published online 27 December 2016)

Gold nanoparticle (GNP) boosted radiation therapy can enhance the biological effectiveness of radiation treatments by increasing the quantity of direct and indirect radiation-induced cellular damage. As the physical effects of GNP boosted radiotherapy occur across energy scales that descend down to $10 \mathrm{eV}$, Monte Carlo simulations require discrete physics models down to these very low energies in order to avoid underestimating the absorbed dose and secondary particle generation. Discrete physics models for electron transportation down to $10 \mathrm{eV}$ have been implemented within the Geant4-DNA low energy extension of Geant4. Such models allow the investigation of GNP effects at the nanoscale. At low energies, the new models have better agreement with experimental data on the backscattering coefficient, and they show similar performance for transmission coefficient data as the Livermore and Penelope models already implemented in Geant4. These new models are applicable in simulations focussed towards estimating the relative biological effectiveness of radiation in GNP boosted radiotherapy applications with photon and electron radiation sources. Published by AIP Publishing. [http://dx.doi.org/10.1063/1.4972191]

\section{INTRODUCTION}

Gold nanoparticles (GNPs) are currently being studied as a means of improving clinical outcomes from radiation therapy. ${ }^{1,2}$ In radiation therapy treatments, nanoparticles are typically high atomic number metal or metal-oxide particles up to a few hundred nanometers in size. Owing to their high atomic number, the particles absorb higher quantities of incident radiation than their surrounding biological medium, increasing the local energy deposition in the region of the nanoparticle. Moreover, following excitation and ionisation inside nanoparticles, large numbers of low energy secondary electrons are produced which may enhance treatment efficiency by increasing the frequency of direct and indirect radiation-induced biological damage.

Over the last decade, experiments have shown that adding heavy nanoparticles to irradiated cells boosts the biological effectiveness of the radiation source used. Across the nanoparticle compositions studied, GNPs show the highest potential to increase the radiosensitivity of cells. ${ }^{3-5} \mathrm{~A}$ dose enhancement from using GNPs in radiotherapy has already been demonstrated using Monte Carlo simulations; ${ }^{6}$ however

\footnotetext{
a) Author to whom correspondence should be addressed. Electronic addresses: dousatsu-univtky@umin.ac.jp and sakata@cenbg.in2p3.fr
}

these simulations are of limited accuracy at micrometer scales, as the continuous physics models used to date break down at small spatial resolutions. ${ }^{7,8}$ As one moves towards more accurate microdosimetric measurements of the impact of GNPs, discrete physics models become necessary as they improve the spatial resolution in simulation. As the physical effects of GNP boosted radiotherapy occur across energy scales that descend down to $10 \mathrm{eV}$, Monte Carlo simulations require discrete physics models down to these very low energies in order to avoid underestimating absorbed dose and secondary particle generation. Currently, very few Monte Carlo codes provide discrete models of electron transport in gold. ${ }^{9,10}$ In this work, we present an implementation of discrete electron transport models in gold inside the Geant 4 Monte Carlo particle transport simulation toolkit. ${ }^{11-13}$

The implementation of GNP transport models in Geant4 provides a valuable tool to the community for GNP-related studies, as it benefits from existing developments within the Geant4-DNA low energy extension of the Geant4 toolkit. Geant4-DNA already implements discrete electron, proton and alpha particles transport models in liquid water ${ }^{14-16}$ as well as modelling the physico-chemical and chemical stages of liquid water radiolysis. ${ }^{17,18}$ These features are available to the medical physics community through Geant 4 itself, as well as the TOPAS ${ }^{19}$ and GATE $^{20}$ packages. 
TABLE I. Summary of implemented physics models for electron transportation.

\begin{tabular}{lccc}
\hline \hline Physics process & Corresponding model & Class name & Energy range \\
\hline Elastic scattering & Relativistic partial wave analysis (ELSEPA) & G4DNAELSEPAElasticModel & $10 \mathrm{eV}-1 \mathrm{GeV}$ \\
Electronic excitation & Dirac B-spline R-matrix + experiment & G4DNADiracRMatrixExcitationModel & $1.14 \mathrm{eV}-1 \mathrm{GeV}$ \\
Plasmon excitation & Quinn model & G4DNAQuinnPlasmonExcitationModel & $9.0 \mathrm{eV}-1 \mathrm{GeV}$ \\
Ionization & Modified relativistic binary encounter Bethe Vriens & G4DNARelativisticlonisationModel & $8.3 \mathrm{eV}-1 \mathrm{GeV}$ \\
Bremsstrahlung & Seltzer and Berger model & G4SeltzerBergerModel & $1 \mathrm{keV}-10 \mathrm{GeV}$ \\
\hline
\end{tabular}

We describe the electron transport models in gold which have been included in Geant4 in Sec. II (Geant4 v10.02.p01 is used throughout this paper). The models for elastic scattering, electronic excitation, plasmon excitation, ionisation, and bremsstrahlung are presented here. We then offer a validation of the models before summarising our results.

\section{DESCRIPTION OF Geant4-DNA PHYSICS MODELS FOR GOLD}

We have implemented new physics models for elastic scattering, electronic and plasmon excitation and ionization within Geant4 for electron transportation in gold. The physics model used for each process is given in Table I, noting that for bremsstrahlung a model from the standard Geant 4 sub-package is used, G4SeltzerBergerModel. ${ }^{21}$ The integrated cross sections for the newly implemented physics models are shown in Figure 1. A similar hybrid approach, where different theories are used to represent the various inelastic processes of the material, has also been employed in the MONSEL code ${ }^{22}$ for secondary electron transport simulations. An alternative approach is the use of a model dielectric response function for the target material. ${ }^{23-26}$ Although the approach based on the dielectric function allows some degree of self-consistency through the restrictions imposed by various sum-rules, it often requires elaborate algorithms for properly deducing the contribution of the individual inelastic processes. ${ }^{27,28}$

The implemented physics models allow electron transport down to $10 \mathrm{eV}$ (shown in grey in Figure 1). This limit comes from the energy limit of elastic scattering. At this level, electron tracks are killed and their energy is deposited locally. For photon transportation, we use the existing

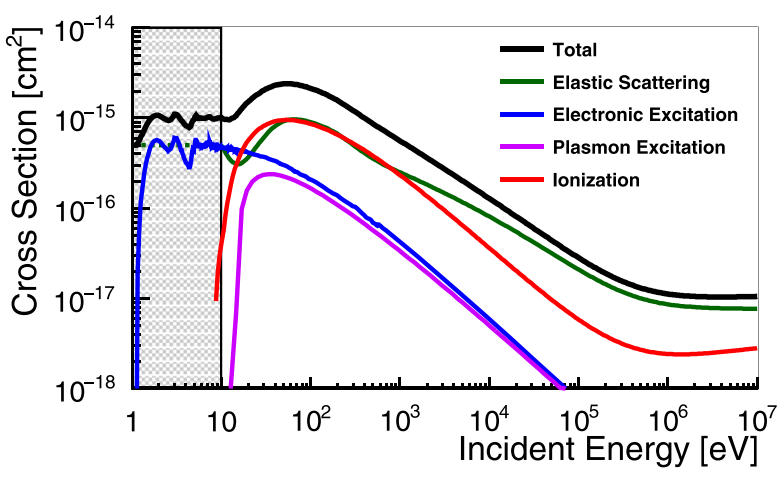

FIG. 1. Integrated electron cross sections as a function of incident electron energy in gold for each Geant4-DNA physics model developed in this work. The cross section for Bremsstrahlung is not shown, as it is significantly lower than the other cross section. All electrons with their energy below $10 \mathrm{eV}$ (shown in grey) are killed and their energy is deposited locally.
Livermore model set already implemented in Geant4 $\left(\right.$ G4EmLivermorePhysics ${ }^{29}$ ). Detailed descriptions of the new models are provided in Secs. II A-II D.

\section{A. Elastic scattering}

The FORTRAN77 code ELSEPA developed by Salvat ${ }^{30}$ was used to calculate scattering angles and integrated cross sections for elastic scattering. ELSEPA calculates the scattering angle in the laboratory frame by a relativistic partialwave method using a local central interaction potential for atoms in both the solid and vapor states, across a wide incident electron energy range $(10 \mathrm{eV}-1 \mathrm{GeV})$. ELSEPA considers the electrostatic interaction potential, electron exchange potential, correlation-polarization potential, and the imaginary absorption potential inside the local central interaction potential. In this study, we calculate differential cross sections from ELSEPA, and then integrate these cross sections in ELSEPA using the default solid state configuration for gold. The default configuration itself is calculated using a bounded atom Muffin-tin model ${ }^{31}$ where a numerical DiracFock distribution has been used to estimate the electron distribution.

\section{B. Electronic excitation}

Four excitation channels are considered in our modelling of the electronic excitation of gold. At lower energies, scaled cross-sections were used based on experimental data. These were complemented by theoretical cross-sections at higher energies. The excitation channels simulated are listed below, along with the upper energy for experimental crosssection data, $E_{\exp } 32,33$

$$
\begin{array}{ll}
\left(5 d^{10} 6 s\right)^{2} S_{1 / 2} \rightarrow\left(5 d^{9} 6 s^{2}\right)^{2} D_{3 / 2} & E_{\exp }<5.8 \mathrm{eV} \\
\left(5 d^{10} 6 s\right)^{2} S_{1 / 2} \rightarrow\left(5 d^{9} 6 s^{2}\right)^{2} D_{5 / 2} & (\text { No Data }) \\
\left(5 d^{10} 6 s\right)^{2} S_{1 / 2} \rightarrow\left(5 d^{10} 6 p\right)^{2} P_{1 / 2} & E_{\exp }<577 \mathrm{eV} \\
\left(5 d^{10} 6 s\right)^{2} S_{1 / 2} \rightarrow\left(5 d^{10} 6 p\right)^{2} P_{3 / 2} & E_{\exp }<577 \mathrm{eV}
\end{array}
$$

This approach of mixing experimental and theoretical cross sections is also used by TRAX. ${ }^{9,10}$ The low-energy cross sections were calculated by scaling relative experimental cross sections to match theoretical cross sections calculated using Dirac B-Spline R-matrices (DBSRMs) ${ }^{32,33}$ which is an extension of the BSR complex ${ }^{34}$ to the fully relativistic Dirac scheme, as the DBSRM calculation differs significantly from experimental data at low energies. The BetheBorn approximation predicts the excitation probability for the S, D, and P states. For the P state, the probability follows 
the analytical function, $\sigma \propto \ln (E) / E$ where $E$ is the incident electron energy. For the $\mathrm{S}$ and $\mathrm{D}$ states, the probability follows $\sigma \propto 1 / E^{2}$. We extend the low energy cross sections using these two analytical formulae when experimental data are not available. No scaled cross sections currently exist based on experimental data for the channel $\left(5 d^{10} 6 s\right)^{2} S_{1 / 2}$ $\rightarrow\left(5 d^{9} 6 s^{2}\right)^{2} D_{5 / 2}$; therefore the DBSRM-based cross section is used for all energies in this channel. All the atomic states used in the DBSRM calculation were calculated using the GRASP2K relativistic atomic structure package. ${ }^{35}$

\section{Plasmon excitation}

Plasmon excitation refers to the excitation of dynamic oscillations in the conduction electrons of a material. In gold, free electrons can lose energy by exciting volume plasmon excitations. Cross-sections and energy losses in volume plasmon excitation are given by Quinn. ${ }^{36}$

The cross section for volume plasmon excitation from the Quinn model can be calculated from the number of atoms per unit volume $n$ and the mean free path $\lambda$ via the equation

$$
\sigma_{\mathrm{PE}}=\frac{1}{n \lambda}=\frac{1}{n} \frac{\hbar \omega_{p}}{2 a_{0} E} \ln \left(\frac{\sqrt{p_{0}^{2}+2 m_{e} \omega_{p} \hbar}-p_{0}}{p-\sqrt{\omega_{p} \hbar\left(p^{2}-2 m_{e}\right)}}\right),
$$

where $E$ is the energy of the incident electron, $\hbar$ is the reduced Planck constant, $a_{0}$ is the Bohr constant, $m_{e}$ is the mass of an electron, and $\omega_{p}$ is the plasmon frequency given by $\sqrt{\left(n_{v} e^{2}\right) /\left(\epsilon_{0} m_{e}\right)}$ where $e$ is the electron charge, $\epsilon_{0}$ is the electrical field constant, $n_{v}$ is the density of valence electrons, and $p$ and $p_{0}$ are the incident electron momentum and Fermi momentum, respectively. The density of valence electrons can be calculated from

$$
n_{v}=\frac{\rho N_{A} N_{v}}{M_{\mathrm{mol}}}
$$

where $\rho$ is the density of the target material, $M_{\text {mol }}$ is its molar mass, $N_{v}$ is the number of valence electrons ( 2 for a complete 6s shell in the ground state of gold), and $N_{A}$ is Avogadro's number. The momenta $p$ and $p_{0}$ are derived from the same formula $p=\sqrt{2 m_{e} E}$. The Fermi momentum $p_{0}$ can be found by replacing $E$ with the Fermi energy $E_{F}$ via $^{9}$

$$
E_{F}=\frac{h^{2}}{8 m_{e}}\left(\frac{3 n_{v}}{\pi}\right)^{2 / 3} \frac{1}{e} .
$$

The energy lost by incident electrons in gold is also calculated theoretically. Following the Quinn model, the energy loss by plasmon excitation can be calculated as:

$$
E_{p}=\hbar \times \omega_{p}=\hbar \times \sqrt{\frac{n_{v} e^{2}}{\epsilon_{0} m_{e}}} .
$$

\section{Ionization and atomic de-excitation}

A modified Relativistic Binary Encounter Bethe Vriens (RBEBV) model was used to calculate the integrated ionization cross section and the energy of electrons ejected during ionization. The RBEBV model is an empirical correction to the Binary Encounter Bethe Vriens model ${ }^{37}$ for high atomic number elements. ${ }^{38,39}$

The energy differential cross section following the RBEBV model $\sigma_{\text {Ioni }}$ can be written in the following form:

$$
\begin{aligned}
\frac{d \sigma_{\text {Ioni }}}{d w}= & \frac{4 \pi a_{0}^{2} \alpha^{4} N}{\left(\beta_{t}^{2}+\left(\beta_{u}^{2}+\beta_{b}^{2}\right) / v\right) 2 b^{\prime}}\left[-\frac{\phi}{t+1}\left(\frac{1}{w+1}+\frac{1}{t-w}\right)\right. \\
& \times \frac{1+2 t^{\prime}}{\left(1+t^{\prime} / 2\right)^{2}}+\frac{1}{(w+1)^{2}}+\frac{1}{(t-w)^{2}}+\frac{b^{\prime 2}}{\left(1+t^{\prime} / 2\right)^{2}} \\
& +\left(\ln \left(\frac{\beta_{t}^{2}}{1-\beta_{t}^{2}}\right)-\beta_{t}^{2}-\ln \left(2 b^{\prime}\right)\right) \\
& \left.\times\left(\frac{1}{(w+1)^{3}}+\frac{1}{(t-w)^{3}}\right)\right]
\end{aligned}
$$

where

$$
\begin{aligned}
t & =T / B, \quad w=W / B, \quad u=U / B, \\
\beta_{t}^{2} & =1-\frac{1}{\left(1+t^{\prime}\right)^{2}}, \quad t^{\prime}=T / m_{e} c^{2}, \\
\beta_{u}^{2} & =1-\frac{1}{\left(1+u^{\prime}\right)^{2}}, \quad u^{\prime}=U / m_{e} c^{2}, \\
\beta_{b}^{2} & =1-\frac{1}{\left(1+b^{\prime}\right)^{2}}, \quad b^{\prime}=B / m_{e} c^{2} .
\end{aligned}
$$

Here, $m_{e}$ is the mass of electrons, $c$ is the speed of light, $T$ is the kinetic energy of the incident electron, $B$ is the bound electron binding energy, $W$ is the ejected electron kinetic energy, $U$ is the bound electron kinetic energy, $a_{0}$ is the Bohr radius, $N$ is the occupation number of the subshell to be ionized, and $\alpha$ is the fine structure constant.

The relativistic form of the Vriens $\phi$ function can be written as

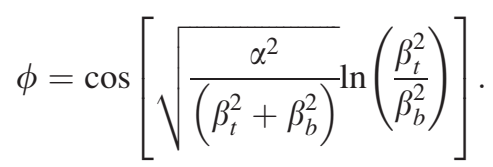

If $\phi=1$, the cross section has no Vriens correction.

We modified the RBEBV calculation by using an empirical parameter $v .^{38}$ This empirical adjustment originally accounted for the high radial kinetic energies encountered in molecular valence shells with high principal quantum numbers $(\geq 3)$, and is set equal to the principal quantum number of the atomic orbital being considered. This parameter has been used across a wide range of chemical molecules, ${ }^{39}$ however here we apply it to an ionic solid. Using this parameter increases the value of the energy differential cross sections and integrated cross sections of outer orbitals, improving the agreement between experimental results ${ }^{40}$ and the overall integrated cross sections in the model.

The theoretical formula for the integrated cross section is given by integration of Eq. (5) formula, namely, 


$$
\begin{aligned}
\sigma_{\text {Ioni }}= & \frac{4 \pi a_{0}^{2} \alpha^{4} N}{\left(\beta_{t}^{2}+\left(\beta_{u}^{2}+\beta_{b}^{2}\right) / v\right) 2 b^{\prime}} \\
& \times\left\{\frac{1}{2}\left[\ln \left(\frac{\beta_{t}^{2}}{1-\beta_{t}^{2}}\right)-\beta_{t}^{2}-\ln \left(2 b^{\prime}\right)\right] \times\left(1-\frac{1}{t^{2}}\right)\right. \\
& \left.+1-\frac{1}{t}-\frac{\ln t}{t+1} \frac{1+2 t^{\prime}}{\left(1+t^{\prime} / 2\right)^{2}} \times \phi+\frac{b^{\prime 2}}{\left(1+t^{\prime} / 2\right)^{2}} \frac{t-1}{2}\right\} .
\end{aligned}
$$

To model atomic de-excitation following ionization, we used the existing atomic de-excitation interface in Geant $4 .^{41}$ This simulates both Auger electrons and fluorescence photons including full cascade relaxation processes. ${ }^{42}$ The deexcitation likelihoods and energies for secondary particles for the 19 inner shells of a gold atom have already been added to the database. To calculate cross sections using the modified RBEBV model and perform atomic de-excitations, we use atomic state parameters, such as binding energies and kinetic energies of orbital electrons from the Livermore Evaluated Atomic Data Library (EADL) database. ${ }^{43}$

\section{VALIDATION OF THE NEW MODELS}

The models described above have been validated against both existing low energy electromagnetic models in Geant4 (specifically the G4EmLivermorePhysics and G4EmPenelopePhysics constructors ${ }^{29}$ ), as well as experimental data where possible. Our first validation tests however are against recommended stopping power and range values from ICRU37 ${ }^{44}$ calculated by ESTAR, ${ }^{45}$ which are a good benchmark for Monte Carlo calculations. The number of secondary particles generated, energy of secondary particles and direction of scattered and secondary particles with respect to the injected particle axis also serve as a good test for physics models. We show secondary particle spectra for electrons and photons with comparison to the Livermore and Penelope models in Geant4. We also show results from simple simulations of electron backscattering from a gold plate, and electron transmission through gold foil. These backscattering and transmission coefficients found from simulation in the new models show a good agreement with both existing data and models.

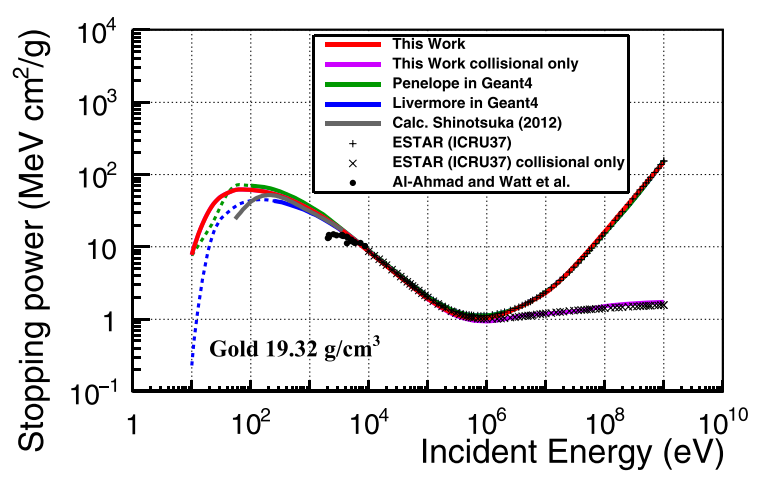

Figure 2 shows average stopping powers and average track lengths for electrons in gold. In general, a good agreement is shown between our models and ICRU37 calculation, as well as the Penelope models at low energy. Regarding average stopping powers, above $10 \mathrm{keV}$, the implemented physics models have good agreement with ICRU37 calculations, experimental data ${ }^{46}$ and existing Geant 4 models. Below this value, the stopping power of the implemented models agrees well with the theoretical model of Shinotsuka. ${ }^{47}$ At such energies (below $100 \mathrm{eV}$ ), experimental measurements are clearly needed to be able to quantify the accuracy of the proposed theoretical models. Since we have not implemented a specific model for bremsstrahlung, we also show the agreement between our models and the ICRU37 stopping powers in the absence of bremsstrahlung at higher energies $\left(\geqslant 10^{6} \mathrm{eV}\right)$. In this scenario, we also see that our models show good agreement with the ICRU recommendations.

For average track lengths, the different models broadly agree across all energies tested, both amongst themselves and with ICRU37 data. Experimental measurements however suggest that the models overestimate the range slightly. ${ }^{48-53}$ In simulation, we calculate the entire track length of incident electrons, including scattering perpendicular to the incident particle direction, to produce a quantity comparable to the ICRU37 recommended values. Experimental ranges underestimate this value as they do not consider contributions to the path length from scattering perpendicular to the initial particle direction. In addition, near $10 \mathrm{eV}$, the transmission range values grow inaccurate as electrons below $10 \mathrm{eV}$ are killed (at $10 \mathrm{eV}$, the average electron range is around $0.4 \mathrm{~nm}$ ).

Accurately simulating the yield and energy of secondary particles generated in GNPs is important in understanding particle track structures inside gold, as well as the biological effects in the surrounding medium, which are dominated by low energy electron interactions. We ran simulations to calculate the spectra of secondary particles generated within bulk gold. Figure 3 shows energy spectra of secondary photons and electrons generated in a $50 \mu \mathrm{m}$ thick gold foil following irradiation by $20 \mathrm{MeV}$ incident electrons. The secondaries come from photoelectric ionization, Compton scattering, pair production, ionization by electron impact, and atomic relaxation following ionization. Bremsstrahlung

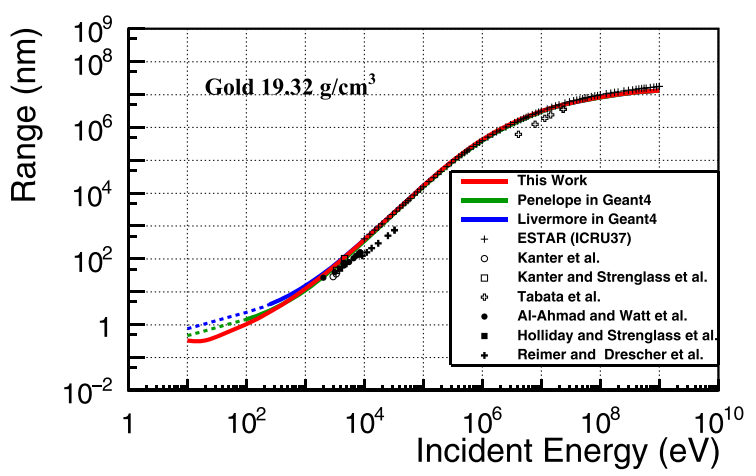

FIG. 2. Comparison of physics models with ESTAR data. Stopping powers (left) and track length (right) are shown alongside ESTAR data and experimental measurements across a range of incident electron energies. Dotted lines are used to show the predictions from the Penelope and Livermore models below their recommended minimum energies. Stopping powers are considered both including and without radiative energy losses from bremsstrahlung. 

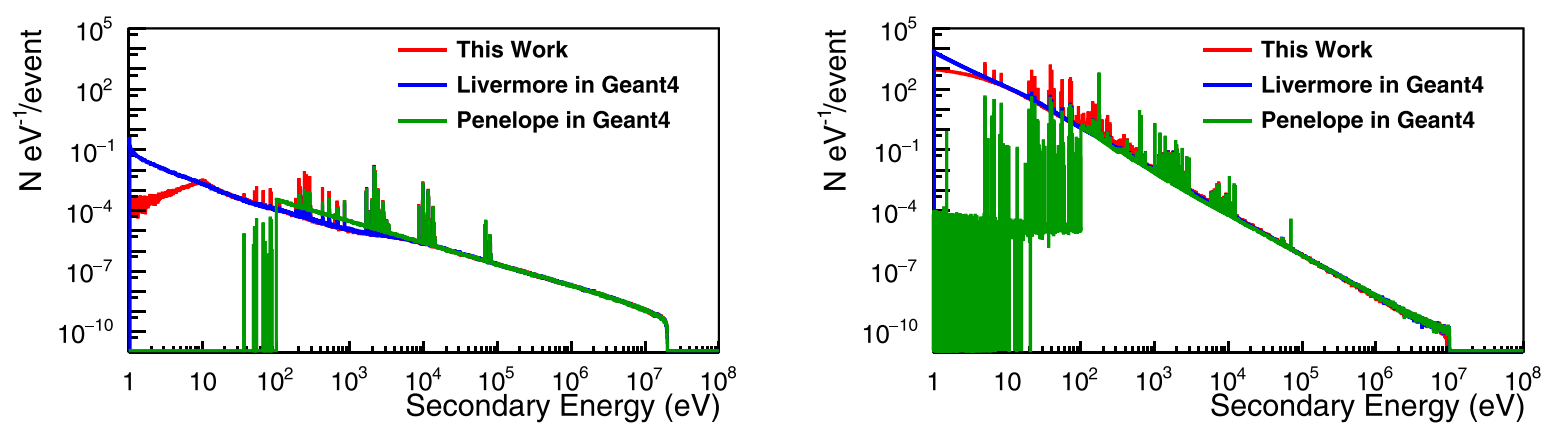

FIG. 3. Energy spectra of secondary particles generated in gold. Both the photon (left) and electron (right) secondary spectra are shown following irradiation by $20 \mathrm{MeV}$ electrons. The production cut for secondary particles is $1 \mathrm{eV}$.

radiation also contributes at high energies to the photon spectrum. For the physics models studied, the majority of secondaries occur between $10 \mathrm{eV}$ and $1 \mathrm{keV}$. It is worth noting the steep drop in secondary production in the Penelope physics models below $100 \mathrm{eV}$ is caused by the model being extended outside its domain of validity.

The backscattering coefficient for electrons incident upon a gold plate, and the transmission coefficient for electrons impacting a thin gold foil provide a good means of validation for electron transport models across a wide energy range. The backscattering coefficient as a function of incident energy is shown in Figure 4. Backscattering simulations are compared with several experimental measurements. ${ }^{54-58}$ In order to provide a fair comparison to experimental data, experimental results are only used when the backscattering coefficient measurement covers close to half or all the possible backscattering solid angle. Two curves are shown for each physics model, the first considering backscattered electrons with energies above $50 \mathrm{eV}$ and the next considering those above $125 \mathrm{eV}$, as the thresholds from the experiment vary between $50-120 \mathrm{eV}$. Especially, at low energies, the new gold physics models have much better agreement with experimental data, compared to the Livermore and Penelope models.

The number of transmitted electrons per unit angle through gold foil was simulated for two different foil

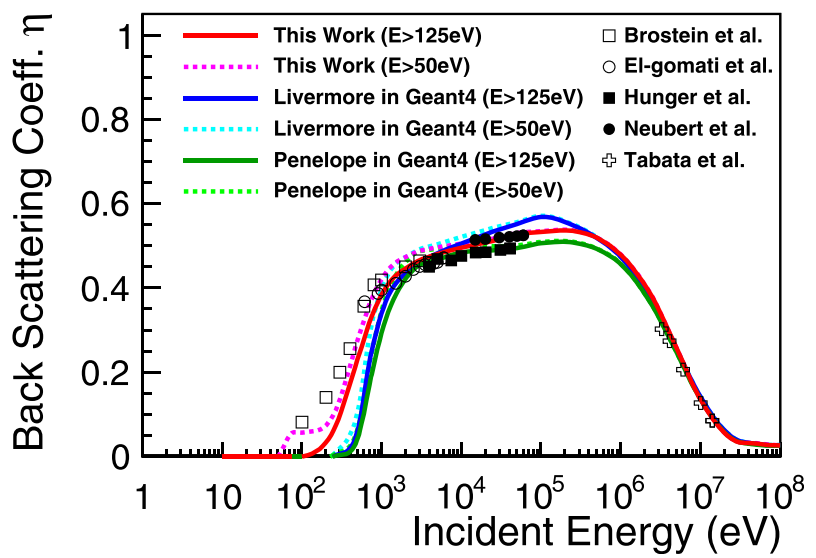

FIG. 4. The backscattering coefficient, $\eta$, from a $5 \mathrm{~cm}$ gold plate in vacuum, as a function of incident electron energy. In this simulation, $50 \mathrm{eV}$ and $125 \mathrm{eV}$ are considered as threshold of backscattered electron counting for fair comparison with experiments. The production cut of secondary particles is $1 \mathrm{eV}$. widths $(9.658 \mu \mathrm{m}$ and $19.296 \mu \mathrm{m})$, using incident electrons with energy $15.7 \mathrm{MeV}$ (Figure 5). The new physics models agree well with the existing physics models in Geant4 for these geometries, as well as with the experimental measurements. ${ }^{59}$

\section{CONCLUSION}

Improved physics models for gold are necessary to better model the impact of GNPs in radiotherapy via Monte Carlo simulations. We implemented new physics models for electron transportation in gold in Geant4 that are applicable down to $10 \mathrm{eV}$. Especially, at low energies, the new models have better agreement with experimental data for the backscattering coefficient, and show roughly similar performance for the transmission coefficient when compared to the Livermore and Penelope models already in Geant 4 . The average track length of the electrons in gold at $10 \mathrm{eV}$ using the new models is around $0.4 \mathrm{~nm}$. These models then allow the simulation of electrons in GNPs down to a few tens of nanometers. The physics models include atomic de-excitation with a full cascade of relaxation processes for gold. Accordingly, these new models are applicable in simulations seeking to measure the biological effect of radiation in GNP-boosted radiotherapy with photon and electron radiation sources.

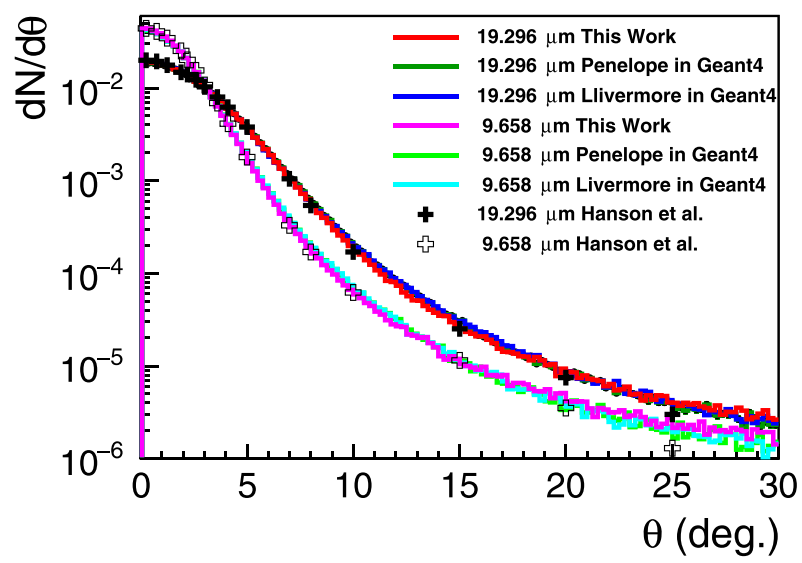

FIG. 5. Distribution of the quantity of transmitted electrons per unit angle as a function of angle (measured from the incident electron direction) for incident $15.7 \mathrm{MeV}$ electrons. Results are shown for electrons transmitted through a gold foil of thickness $9.658 \mu \mathrm{m}$ and $19.296 \mu \mathrm{m}$. 


\section{ACKNOWLEDGMENTS}

The authors would like to express their gratitude to Pr. Francesc Salvat (Barcelona University, Spain) who very kindly provided us with the ELSEPA code. This work is funded by the University of Bordeaux, via the 2015 international post-doctoral fellowship program, for the "NanoBoost" project. The work is also supported by "the France-Japan Particle Physics Laboratory (FJPPL)" international associated laboratory (CNRS/KEK) and from the Greece-France "Projet International de Cooperation Scientifique (PICS)" \#7340. This work is partially supported by the Australian Research Council, ARC DP ID DP170100967.

${ }^{1}$ D. M. Herold et al., "Gold microspheres: A selective technique for producing biologically effective dose enhancement," Int. J. Radiat. Biol. 76(10), 1357-1364 (2000).

${ }^{2}$ J. F. Hainfeld et al., "The use of gold nanoparticles to enhance radiotherapy in mice," Phys. Med. Biol. 49(18), N309-N315 (2004).

${ }^{3}$ S. Jain et al., "Gold nanoparticles as novel agents for cancer therapy," Br. J. Radiol. 85(1010), 101-113 (2012).

${ }^{4}$ D. B. Chithrani et al., "Gold nanoparticles as radiation sensitizers in cancer therapy," Radiat. Res. 173(6), 719-728 (2010).

${ }^{5} \mathrm{R}$. Iezzi et al., "Dendrimer-based targeted intravitreal therapy for sustained attenuation of neuroinflammation in retinal degeneration," Biomaterials 33(3), 979-988 (2012).

${ }^{6} \mathrm{~S}$. X. Zhang et al., "Quantifying tumor-selective radiation dose enhancements using gold nanoparticles: A Monte Carlo simulation study," Biomed. Microdevices 11(4), 925-933 (2009).

${ }^{7} \mathrm{M}$. A. Bernal et al., "An investigation on the capabilities of the PENELOPE MC code in nanodosimetry," Med. Phys. 36, 620 (2009).

${ }^{8} \mathrm{~J}$. M. Fernandez-Varea et al., "Limitations (and merits) of PENELOPE as a track-structure code,” Int. J. Radiat. Biol. 88, 66-70 (2012).

${ }^{9} \mathrm{C}$. Walzlein, "Nanometer scale description of electron transport and damage in condensed media using the TRAX Monte Carlo code," Ph.D. thesis, Technische Universitat Darmstadt, 2014.

${ }^{10} \mathrm{C}$. Walzlein et al., "Simulations of dose enhancement for heavy atom nanoparticles irradiated by protons," Phys. Med. Biol. 59, 1441-1458 (2014).

${ }^{11}$ GEANT4 Collaboration, "Geant4-A simulation toolkit," Nucl. Instrum. Methods Phys. Res., Sect. A 506, 250-303 (2003).

${ }^{12}$ GEANT4 Collaboration, "Geant4 developments and applications," IEEE Trans. Nucl. Sci. 53(1), 270-278 (2006).

${ }^{13}$ Geant4 Collaboration, "Recent developments in Geant4," Nucl. Instrum. Methods Phys. Res., Sect. A 835, 186-225 (2016).

${ }^{14}$ S. Incerti et al., "The Geant4-DNA project," Int. J. Modell. Simul. Sci. Comput. 1, 157-178 (2010).

${ }^{15} \mathrm{~S}$. Incerti et al., "Comparison of Geant 4 very low energy cross section models with experimental data in water," Med. Phys. 37, 4692-4708 (2010).

${ }^{16} \mathrm{M}$. A. Bernal et al., "Track structure modeling in liquid water: A review of the Geant4-DNA very low energy extension of the Geant 4 Monte Carlo simulation toolkit," Phys. Med. 31, 861-874 (2015).

${ }^{17}$ M. Karamitros et al., "Modeling radiation chemistry in the Geant4 toolkit," Prog. Nucl. Sci. Technol. 2, 503-508 (2011).

${ }^{18} \mathrm{M}$. Karamitros et al., "Diffusion-controlled reactions modeling in Geant4DNA,” J. Comput. Phys. 274, 841-882 (2014).

${ }^{19}$ J. Perl et al., "TOPAS: An innovative proton Monte Carlo platform for research and clinical applications," Med. Phys. 39(11), 6818-6837 (2012).

${ }^{20}$ G. Santin et al., "GATE: A Geant4-based simulation platform for PET, SPECT integrating movement and time management," IEEE Trans. Nucl. Sci. 50, 1516-1521 (2003).

${ }^{21}$ J. Allison et al., "Geant4 electromagnetic physics for high statistics simulation of LHC experiments," J. Phys.: Conf. Ser. 396, 022013 (2012).

${ }^{22}$ J. R. Lowney et al., "Monte Carlo simulation of scanning electron microscope signals for lithographic metrology," Scanning 18, 301-306 (1996).

${ }^{23}$ A. Akkerman et al., "Ion and electron track structure and its effects in silicon: Model and calculations," Nucl. Instrum. Methods Phys. Res., Sect. B 227, 319-336 (2005)
${ }^{24}$ J. M. Fernandez-Varea et al., "Inelastic scattering of electrons in solids from a generalized oscillator strength model using optical data photoelectric data," J. Phys.: Condens. Matter 5, 3593-3610 (1993).

${ }^{25}$ R. Garcia-Molina et al., "Inelastic scattering and energy loss of swift electron beams in biologically relevant materials," Surf. Interface Anal. 49, 11-17 (2017).

${ }^{26} \mathrm{H}$. Nikjoo et al., "Radiation track, DNA damage and response-A review,” Rep. Prog. Phys. 79, 116601 (2016).

${ }^{27}$ I. Kyriakou et al., "Improvements in Geant4 energy-loss model and the effect on low-energy electron transport in liquid water," Med. Phys. 42, 3870-3876 (2015).

${ }^{28}$ I. Kyriakou et al., "The impact of new Geant4-DNA cross section models on electron track structure simulations in liquid water," J. Appl. Phys. 119, 194902 (2016).

${ }^{29} \mathrm{~V}$. Ivantchenko et al., "Recent improvements in Geant4 electromagnetic physics models and interfaces," Prog. Nucl. Sci. Technol. 2, 898-903 (2011).

${ }^{30} \mathrm{~F}$. Salvat et al., "ELSEPA-Dirac partial-wave calculation of elastic scattering of electrons and positrons by atoms, positive ions and molecules," Comput. Phys. Commun. 165, 157-190 (2005).

${ }^{31} \mathrm{Ch}$. Kittel et al., Introduction to Solid State Physics, 5th ed. (John Wiley and Sons, New York, 1976).

${ }^{32} \mathrm{M}$. Maslov et al., "Electron-impact excitation of the $\left(5 \mathrm{~d}^{10} 6 \mathrm{~s}\right)^{2} \mathrm{~S}_{1 / 2} \rightarrow$ $\left(5 \mathrm{~d}^{10} 6 \mathrm{p}\right)^{2} \mathrm{P}_{1 / 2,3 / 2}$ resonance transitions in gold atoms," Phys. Rev. A 77, 062711 (2008).

${ }^{33} \mathrm{O}$. Zatsarinny et al., "Electron-impact excitation of the $\left(5 \mathrm{~d}^{10} 6 \mathrm{~s}\right)^{2} \mathrm{~S}_{1 / 2} \rightarrow$ $\left(5 \mathrm{~d}^{9} 6 \mathrm{p}^{2}\right)^{2} \mathrm{D}_{5 / 2,3 / 2}$ transitions in gold atoms," Phys. Rev. A 78, 042713 (2008).

${ }^{34}$ O. Zatsarinny, "BSR: B-spline atomic R-matrix codes," Comput. Phys. Commun. 174, 273 (2006).

${ }^{35} \mathrm{P}$. Jonsson et al., "The grasp2K relativistic atomic structure package," Comput. Phys. Commun. 177, 597 (2007).

${ }^{36} \mathrm{~J}$. J. Quinn et al., "Range of excited electrons in metals," Phys. Rev. 126, 1453 (1962)

${ }^{37}$ M. Guerra et al., "Single differential electron impact ionization cross sections in the binary-encounter-Bethe approximation for the low binding energy regime," J. Phys. B: At., Mol. Opt. Phys. 48, 185202 (2015).

${ }^{38} \mathrm{~K}$. K. Irikura et al., "Electron-impact total ionization cross-sections of the chlorofluoromethanes," Int. J. Mass Spectrom. 222, 189 (2003).

${ }^{39}$ S. Edel, "Modelisation du transport des photons et des electrons," Ph.D. thesis, Univ. Toulouse III, 2006.

${ }^{40} \mathrm{~J}$. M. Schroeer et al., "Electron impact ionization cross sections of $\mathrm{Cu}$ and Au between 40 and $250 \mathrm{eV}$, and the velocity of evaporated atoms," J. Chem. Phys. 58, 5135 (1973).

${ }^{41}$ A. Mantero et al., "PIXE simulation in Geant4," X-Ray Spectrom. 40, 135-140 (2011).

${ }^{42}$ S. Incerti et al., "Simulation of Auger electron emission from nanometersize gold targets using the Geant4 Monte Carlo simulation toolkit," Nucl. Instrum. Methods Phys. Res., Sect. B 372, 91-101 (2016).

${ }^{43}$ S. T. Perkins et al., Tables and Graphs of Atomic Subshell and Relaxation Data Derived from the LLNL Evaluated Atomic Data Library (EADL). $Z=1-100$ (Lawrence Livermore National Lab, CA, 1991), Vol. 30.

${ }^{44}$ ICRU, "Stopping powers for electrons and positrons," Report No. 37, International Commission on Radiation Units and Measurements, Bethesda, MD, 1984.

${ }^{45}$ M. J. Berger et al., "ESTAR, PSTAR, and ASTAR: Computer programs for calculating stopping-power and range tables for electrons, protons, and helium ions," NISTIR 4999, National Institute of Standards and Technology, Gaithersburg, MD, 1992.

${ }^{46}$ R. K. Batra et al., "Approximate stopping power law of electrons and positrons,” Nucl. Instrum. Methods 109, 565 (1973).

${ }^{47} \mathrm{H}$. Shinotsuka et al., "Calculations of electron stopping powers for 41 elemental solids over the $50 \mathrm{eV}$ to $30 \mathrm{keV}$ range with the full Penn algorithm," Nucl. Instrum. Methods Phys. Res., Sect. B 270, 75 (2012).

${ }^{48} \mathrm{H}$. Kanter et al., "Electron scattering by thin foils for energies below 10 keV," Phys. Rev. 121, 461 (1961).

${ }^{49} \mathrm{H}$. Kanter et al., "Interpretation of range measurements for kilovolt electrons in solids," Phys. Rev. 126, 620 (1962).

${ }^{50} \mathrm{~K}$. O. Al-Ahmad et al., "Stopping powers and extrapolated ranges for electrons (1-10 keV) in metals," J. Phys. D: Appl. Phys. 16, 2257-2267 (1983)

${ }^{51} \mathrm{~T}$. Tabata et al., "Extrapolated and projected ranges of 4- to $24-\mathrm{MeV}$ electrons in elemental materials," J. Appl. Phys. 42, 3361 (1971).

${ }^{52} \mathrm{~L}$. Reimer et al., "Secondary electron emission of 10-100 keV electrons from transparent FDMS of A1 and Au," J. Phys. D: Appl. Phys. 10, 805-815 (1977). 
${ }^{53}$ J. E. Holliday et al., "New method for range measurements of low-energy electrons in solids," J. Appl. Phys. 30, 1428 (1959).

${ }^{54} \mathrm{H}$. J. Hunger et al., "Measurements of the electron backscattering coefficient for quantitative EPMA in the energy range of 4 to $40 \mathrm{keV}$," Phys. Status Solidi A 56, K45 (1979).

${ }^{55} \mathrm{G}$. Neuber et al., "Backscattering coefficient measurements of 15 to 60 $\mathrm{keV}$ electrons for solids at various angles of incidence," Phys. Status Solidi A 59, 35 (1980).
${ }^{56} \mathrm{~T}$. Tabata et al., "Backscattering of electrons from 3.2 to $14 \mathrm{MeV}$," Phys. Rev. 162, 336 (1967).

${ }^{57}$ M. M. El-Gomati et al., "Theory experiment comparison of the electron backscattering factor from solids at low electron energy $(250-5,000 \mathrm{eV})$," Scanning 30, 2-15 (2008).

${ }^{58}$ M. I. Bronstein et al., Vtorichnaya Elektronnaya Emissiya (Nauka, Moskva, 1969), p. 340.

${ }^{59}$ A. O. Hanson et al., "Measurement of multiple scattering of $15.7-\mathrm{Mev}$ electrons," Phys. Rev. 84, 634 (1951). 